\title{
Dielectric properties of single wall carbon nanotubes-based gelatin phantoms
}

\author{
M. M. Altarawneh ${ }^{*}$, G. A. Alharazneh* and O. Y. Al-Madanat ${ }^{\dagger}$, \\ *Department of Physics, Mutah University \\ Mutah, Alkarak 61710, Jordan \\ ${ }^{\dagger}$ Department of Chemistry, Mutah University, Mutah, Alkarak 61710, Jordan \\ Institute for Technical Chemistry Gottfried Wilhelm Leibniz Universität \\ Hannover Callinstraße 5, 30167 Hannover, Germany \\ \$moaz@mutah.edu.jo
}

Received 18 February 2018; Revised 21 March 2018; Accepted 26 March 2018; Published 17 April 2018

\begin{abstract}
In this work, we report the dielectric properties of Single wall Carbon Nanotubes (SWCNTs)-based phantom that is mainly composed of gelatin and water. The fabricated gelatin-based phantom with desired dielectric properties was fabricated and doped with different concentrations of SWCNTs (e.g., $0 \%, 0.05 \%, 0.10 \%, 0.15 \%, 0.2 \%, 0.4 \%$ and $0.6 \%$ ). The dielectric constants (real $\varepsilon^{\prime}$ and imaginary $\left.\varepsilon^{\prime \prime}\right)$ were measured at different positions for each sample as a function of frequency $(0.5-20 \mathrm{GHz})$ and concentrations of SWCNTs and their averages were found. The Cole-Cole plot $\left(\varepsilon^{\prime}\right.$ versus $\left.\varepsilon^{\prime \prime}\right)$ was obtained for each concentration of SWCNTs and was used to obtain the static dielectric constant $\varepsilon_{s}$, the dielectric constant at the high limit of frequency $\varepsilon_{\infty}$ and the average relaxation time $\tau$. The measurements showed that the fabricated samples are in good homogeneity and the SWCNTs are dispersed well in the samples as an acceptable standard deviation is achieved. The study showed a linear increase in the static dielectric constant $\varepsilon_{s}$ and invariance of the average relaxation time $\tau$ and the value of $\varepsilon_{\infty}$ at room temperature for the investigated concentrations of SWCNTs.
\end{abstract}

Keywords: Single-wall carbon nanotubes; dielectric; relaxation time; Cole-Cole model.

\section{Introduction}

Carbon nanotube-based materials because of their high aspect ratio hold the promise of providing new revolutionary technological applications in many fields. ${ }^{1,2}$ The interaction of electromagnetic radiation (e.g., microwaves) with carbon nanotubes-based materials is one of the active research areas that has potential technological applications in industry and medical fields. ${ }^{3-6}$ Recently, there were many studies focused on the microwave effects in carbon nanotubes-based materials and its medical applications aiming to diagnose and cure diseases (e.g., cancer disease). ${ }^{7-11}$ In the medical research field area, to achieve the later aims, there is a high demand on the fabrication and characterization of phantoms that represent electrical properties of Human organ's tissues (e.g., breast tissues).

Usually, the desired phantoms that represent the dielectric properties of specific tissues in human body after being well characterized are doped with different types of carbon nanotubes or nanomaterials. For example, in recent publications (e.g., by Mashal et al. ${ }^{13}$ ), the microwaves' heating effect in such phantoms has been studied for different concentrations of single wall carbon nanotubes (SWCNTs) aiming for breast cancer diagnosis and cure. ${ }^{12-16}$ Most studies in this field focus on the fabrication of phantoms with real and imaginary dielectric constants $\left(\varepsilon^{\prime}, \varepsilon^{\prime \prime}\right)$ that are similar to a desired Human tissue in the frequency range of interest. ${ }^{17-19}$ However, other dielectric parameters like static dielectric constant $\varepsilon_{s}$, the dielectric constant at the high limit frequency $\varepsilon_{\infty}$ and the average relaxation time $\tau$ have drawn less attention.

Monitoring the evolution of the dielectric properties as carbon nanotubes or other dopants introduced to phantoms is crucial for studying and optimizing technological applications. Moreover, such monitoring can be used as an indication of dispersion of carbon nanotubes in phantoms.

In this work, the dielectric parameters of $\varepsilon_{s}, \varepsilon_{\infty}$ and $\tau$ will be investigated as a function of SWCNTs concentration using the Cole-Cole model. The chosen phantom in this study is made mainly out of water and gelatin and no oil in its content (oil is usually used to produce phantoms that mimic tissues with low water percentage ${ }^{17}$ ). This study was conducted in the frequency range of $0.5 \mathrm{GHz}$ to $20 \mathrm{GHz}$ and at room temperature around $25^{\circ} \mathrm{C}$.

In the rest of this paper, four sections will be presented as follows: in Sec. 2, the theoretical background of the dielectric properties of materials will be discussed, while in Sec. 3, a description of the sample preparation will be presented along with a description of the dielectric parameters measurement setup, Sec. 4 will go over the results and discuss them thoroughly, at the end, summary of the main conclusions will be presented.

This is an Open Access article published by World Scientific Publishing Company. It is distributed under the terms of the Creative Commons Attribution 4.0 (CC-BY) License. Further distribution of this work is permitted, provided the original work is properly cited. 


\section{Theory}

There are many theoretical models that can be used to explain the dielectric properties of materials depending on the number of reorientational processes taking place in the material when an oscillating electric field is applied. ${ }^{20}$ For materials with single reorientational process, the Debye relaxation model can be utilized to fully obtain the dielectric parameters of a material. The mathematical relation of Debye model which relates the complex dielectric constant $\left(\varepsilon^{*}(\omega)=\right.$ $\left.\varepsilon^{\prime}-i \varepsilon^{\prime \prime}\right)$ with frequency of electric field can be rewritten as

$$
\varepsilon^{*}(\omega)=\varepsilon_{\infty}+\frac{\varepsilon_{s}-\varepsilon_{\infty}}{1+i \omega \tau},
$$

where $\varepsilon_{\infty}$ is the permittivity measured at the high frequency limit of a polarizable entity when it is unable to respond to the electric field, $\varepsilon_{s}$ is the limiting low frequency permittivity, $\omega$ is the angular frequency, $i$ is $\sqrt{-1}$ and $\tau$ is the time characteristic of the response or relaxation time. For a material or a composite that has $N$ characteristic responses, Debye formulation can be written as

$$
\varepsilon^{*}(\omega)=\varepsilon_{\infty}+\sum_{m=1}^{N} \frac{\Delta \varepsilon_{m}}{1+i \omega \tau_{m}} .
$$

Here, $\Delta \varepsilon_{m}$ is the dielectric strength of the $m$ th response and $N$ is the order (number of terms) of Debye's relation. Plotting $\varepsilon^{\prime \prime}$ with $\varepsilon^{\prime}$ in the complex plane yields a semicircle that its center is on the horizontal axis at $\left(\frac{\varepsilon_{s}+\varepsilon_{\infty}}{2}, 0\right)$ with radius of $\frac{\varepsilon_{s}-\varepsilon_{\infty}}{2}$. However, for systems that have skewed semicircle, one can use the Havriliak-Negami model which is given as follows:

$$
\varepsilon^{*}(\omega)=\varepsilon_{\infty}+\frac{\left(\varepsilon_{s}-\varepsilon_{\infty}\right)}{\left(1+(i \omega \tau)^{1-\alpha}\right)^{\beta}} .
$$

A special case of the Havriliak-Negami formula is the ColeCole formula when $1 \geq \alpha>0$ and $\beta=1$ and the ColeDavidson formula with $\alpha=1$ and $1 \geq \beta>0$. When using the Cole-Cole formula (assuming $1-\alpha=n$ ), the real and imaginary dielectric constants can be written, respectively, as $^{20,21}$ :

$$
\begin{array}{r}
\varepsilon^{\prime}=\varepsilon_{\infty}+\left(\varepsilon_{s}-\varepsilon_{\infty}\right) \frac{1+(\omega \tau)^{n} \cos \left(\frac{n \pi}{2}\right)}{1+2(\omega \tau)^{n} \cos \left(\frac{n \pi}{2}\right)+(\omega \tau)^{2 n}}, \\
\varepsilon^{\prime \prime}=\left(\varepsilon_{s}-\varepsilon_{\infty}\right) \frac{(\omega \tau)^{n} \sin \left(\frac{n \pi}{2}\right)}{1+2(\omega \tau)^{n} \cos \left(\frac{n \pi}{2}\right)+(\omega \tau)^{2 n}} .
\end{array}
$$

Using Eqs. (4) and (5), the relation that relates $\varepsilon^{\prime \prime}$ with $\varepsilon^{\prime}$ is written as

$$
\begin{gathered}
\left(\varepsilon^{\prime}-\frac{\varepsilon_{s}+\varepsilon_{\infty}}{2}\right)^{2}+\left(\varepsilon^{\prime \prime}+\frac{\left(\varepsilon_{s}-\varepsilon_{\infty}\right)}{2} \cot (n \pi / 2)\right)^{2} \\
=\left(\frac{\varepsilon_{s}-\varepsilon_{\infty}}{2} \csc (n \pi / 2)\right)^{2} .
\end{gathered}
$$

The values of $\varepsilon_{s}, \varepsilon_{\infty}$ and $n$ can be extracted by fitting the experimental data with Eq. 6 . The average relaxation time $\tau$ can be obtained by finding the angular frequency $\omega$ at which the $\varepsilon^{\prime \prime}$ reaches its maximum value, so that $\tau=1 / \omega$. The angular frequency is equal to $2 \pi f$, where $f$ is the frequency in units of $\mathrm{Hz}$.

\section{Materials and Methods}

In this part of the paper, the detailed procedure for the fabrication of the chosen phantom doped with SWCNTs and the experimental setup for measuring the dielectric properties are presented.

\subsection{Fabrication of SWCNT-based Phantoms}

In order to fabricate a phantom with desired dielectric properties, a simple procedure is considered to fabricate the phantom as in Refs. 17 and 22. In Ref. 22, many phantoms were made with the range of dielectric properties by varying the gelatin and oil percentage in the phantoms. However, in the current study to investigate the effect of SWCNTs addition only, it has been chosen to fix the concentration of oil in the samples to be $0 \%$ and to use water only. The following steps describe in details the fabrication procedure of the phantom and the addition of SWCNTs:

(i) Starting with a $0.129 \mathrm{~g}$ of $p$-toluic acid measured using analytical balance and mixed with $6.43 \mathrm{~mL}(5.1645 \mathrm{~g})$ of $n$-propanol, then the solution was heated with stirring in water bath $\left(50^{\circ} \mathrm{C}\right)$ until all the $p$-toluic powder is dissolved and the solution becomes clear.

(ii) Then, a $122.73 \mathrm{~mL}$ of deionized water was added to the prepared solution at room temperature, which will form a turbid solution.

(iii) A $21.965 \mathrm{~g}$ of 200 bloom gelatin derived from bovine skin (Sigma Aldrich) was added to the turbid solution portion by portion with stirring at room temperature until the entire gelatin was wetted.

(iv) The beaker with contents was wrapped with many layers of plastic film (an available commercial film is used) and held in a water bath of $\sim 90^{\circ} \mathrm{C}$.

(v) The mixture was heated at $90^{\circ} \mathrm{C}$ in a water bath until it became transparent and no air bubbles were suspended.

(vi) The beaker was removed from the water bath and the mixture was stirred to produce uniformity, then any air bubbles on the surface are removed.

(vii) Partially, the beaker immersed in a water bath of cool water $\left(20-25^{\circ} \mathrm{C}\right)$ and stirred until it cools to $50^{\circ} \mathrm{C}$, then removed from the water bath and the desired mass of SWCNTs is added (supplied by Cheap Tubes Co., with length of $0.5-2.0 \mu \mathrm{m}$, purity $>90 \mathrm{wt} . \%$ outer diameter $1-4 \mathrm{~nm}$, MWCNTs content $<5 \mathrm{wt} . \%$, ash $<1.5$ wt. \%).

(viii) The mixture then stirred well until the carbon nanotube distributed uniformly within the solution and then it was sonicated in ultrasonic bath for $15 \mathrm{~min}$ at $50^{\circ} \mathrm{C}$. 
Table 1. The percentage of starting materials in the prepared phantom.

\begin{tabular}{lcccc}
\hline$p$-Toluic acid & $n$-Propanol & Deionized water & Gelatin & Formaldehyde \\
\hline $0.0861 \%$ & $3.443 \%$ & $81.643 \%$ & $14.643 \%$ & $0.1849 \%$ \\
\hline
\end{tabular}

(ix) The emulsion was stirred using a hot plate with stirrer for few $\min$ at $40^{\circ} \mathrm{C}$ and a $0.637 \mathrm{~g}$ of formaldehyde solution 37 wt. \% in $\mathrm{H}_{2} \mathrm{O}$ was added using a micropipette during the stirring step. Finally, continuing stirring the emulsion on the hot plat stirrer without heating until it cools to $25^{\circ} \mathrm{C}$ and then the formed material was poured in a small glass beaker for cooling and solidification. The percentage of the materials in the phantom is summarized in Table 1.

The flowchart in Fig. 1 summarizes the main steps to fabricate the phantom doped with the SWCNTs in this work. The phantoms were molded in cylindrical cups with a flat bottom with a diameter of $4.0 \mathrm{~cm}$ and a height of $3.0 \mathrm{~cm}$. The fabricated phantoms with different concentrations of SWCNTs were left to cure for three days while covered with plastic film to prevent being exposed to air.

\subsection{Dielectric properties measurements}

In this study, the open-ended coaxial probe method was utilized to study the samples dielectric properties. ${ }^{23-25}$ The dielectric properties of the phantom were studied using a Network Analyzer (model E5071C) with a dielectric probe (open coaxial end probe - performance probe) both made by Keysight Company as demonstrated in Fig. 2. Before performing the measurement, the dielectric probe needs to be calibrated by measuring a short conducting plate and by measuring deionized water at room temperature. The calibration and measurement process were all performed at room temperature $\left(\sim 25^{\circ}\right) \mathrm{C}$.

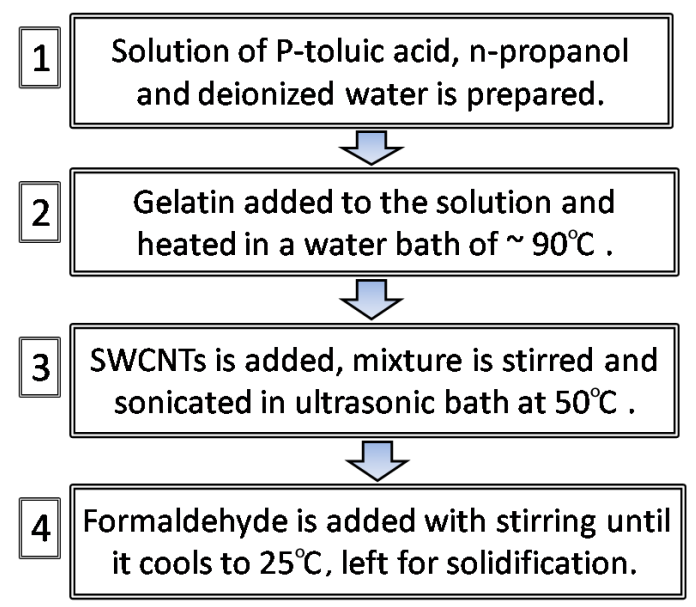

Fig. 1. The main steps of preparing the phantom that is doped with SWCNTs.

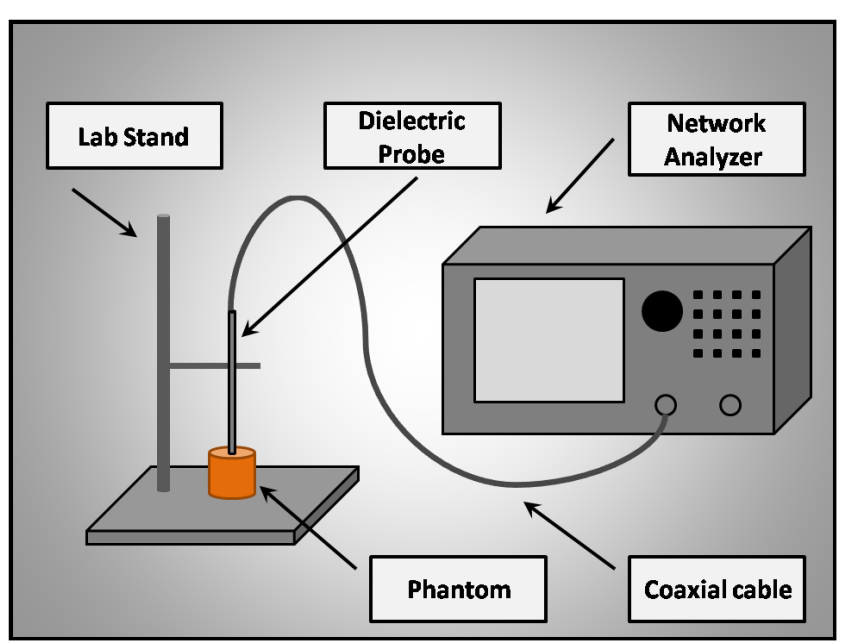

Fig. 2. The schematic diagram of the measurement setup used for studying the dielectric properties of the fabricated phantom.

In order to measure the dielectric properties that represent accurately the phantoms, the dielectric constants (real and imaginary) were measured at five different positions at the surface of the sample and their average is plotted with frequency.

\section{Results and Discussion}

The experimental setup for measuring the dielectric parameters presented in the previous section was used to measure the real and imaginary dielectric constants as a function of frequency as demonstrated in Fig. 3. It is clear in Fig. 3 that as the SWCNTs' concentration is increased, the real and imaginary dielectric constants are increased too in the

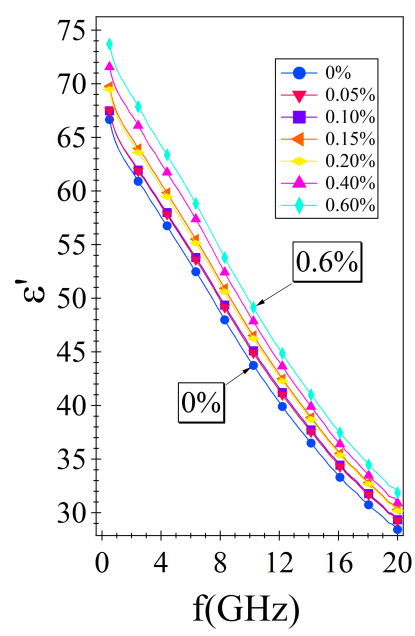

(a)

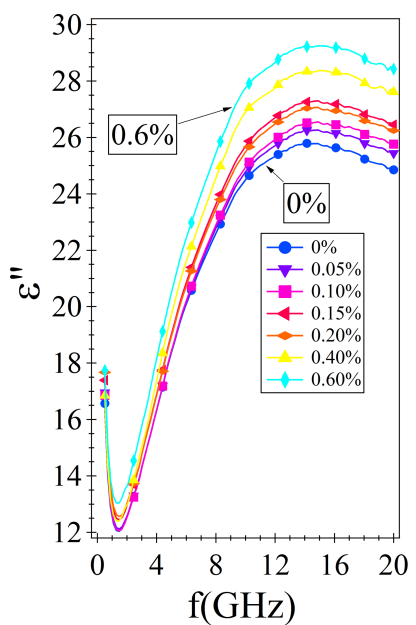

(b)
Fig. 3. (a) The real dielectric constant $\varepsilon^{\prime}$ and (b) the imaginary dielectric constant $\varepsilon^{\prime \prime}$ as a function of frequency for different concentrations of SWCNTs (each trace is the average of five measurements). 
frequency range of $0.5 \mathrm{GHz}$ to $20 \mathrm{GHz}$ at room temperature. However, while the real dielectric constant is increased over the entire frequency range of $0.5 \mathrm{GHz}$ to $20 \mathrm{GHz}$ by the same amount approximately, the increase in the imaginary dielectric constant is larger in the high frequency range.

For the low frequency range $(0.5 \mathrm{GHz}$ to $1.5 \mathrm{GHz})$, the imaginary dielectric constant is attributed to the ionic conductivity generated from salt residues from gelatin powder during fabrication. ${ }^{26}$ The relation $\left(\varepsilon_{\mathrm{dc}}^{\prime \prime}=\sigma_{\mathrm{dc}} / \varepsilon_{0} \omega\right)$ provides the imaginary dielectric constant due to the contribution of ionic conductivity which is decreasing as the frequency is increased $\left(\sigma_{\mathrm{dc}}\right.$ is the dc conductivity at the low frequency limit).

Since the measurement was performed at five different locations and their average was found, it is important to calculate the standard deviation (SD) to investigate the variance of $\varepsilon^{\prime}$ and $\varepsilon^{\prime \prime}$ from one location to another. The variation in the samples quality was investigated by monitoring the SD of the measurements for the $l$ locations calculated for each concentration of SWCNTs using the following relation:

$$
\mathrm{SD}=\sqrt{\frac{\sum_{i=1}^{l}\left(\varepsilon_{i}-\varepsilon\right)^{2}}{l-1}},
$$

where $\varepsilon$ is the average dielectric constant for the $l$ locations at specific frequency. Figure 4 shows the calculated SD plotted for each SWCNTs concentration as a function of frequency for the real and imaginary dielectric constants. The values of the calculated SD are found to be around the typical accuracy of the used dielectric probe as provided by manufacturer $\Delta \varepsilon^{\prime}=\mp 0.05\left|\varepsilon^{*}\right|$ and $\Delta \varepsilon^{\prime \prime}=\mp 0.05\left|\varepsilon^{*}\right|{ }^{27}$ The reasonably small values of calculated SD are a strong indication of a good homogeneity of the samples and an indication of a good dispersion of SWCNTs in the phantom. ${ }^{28}$



(a)



(b)
Fig. 4. The calculated SD for the measurements of (a) the real dielectric constant and (b) the imaginary dielectric constant as a function of frequency for all of the used SWCNTs concentrations.

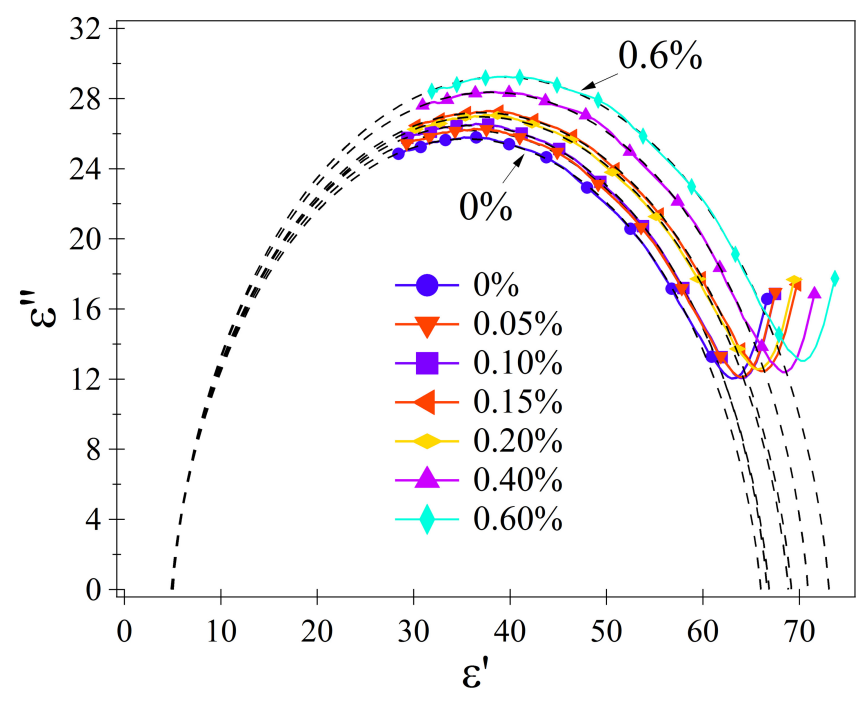

Fig. 5. The imaginary dielectric constant on the vertical axis as plotted with the real dielectric constant in the horizontal axis for different concentrations of SWCNTs that have been used in this study.

Moreover, in Fig. 4 one, can observe that as the applied frequency is increased; the obtained SD for the real dielectric constant is found to decrease for most concentrations and to increase for the imaginary dielectric constant.

The Cole-Cole plot is constructed for this study by plotting the imaginary dielectric constant on $y$-axis with real dielectric constant on $x$-axis as in Fig. 5. Using Eq. (6), the data in Fig. 5 can be fit and the dielectric parameters can be estimated for each SWCNTs concentration. In this study, it has been found out that to obtain good fit for the data, the value of $\alpha$ should be converging to 0.1 for all the concentrations of SWCNTs. The invariance of the value of the exponent $\alpha$ with SWCNTs concentration addition means that SWCNTs addition does not change the overall shape of the Cole-Cole plot. From the Cole-Cole plot fitting, the value of $\varepsilon_{\infty}$ is directly estimated to be around 5 (close to $\varepsilon_{\infty}$ of pure water at room temperature) and invariant with the addition of SWCNTs (see $\varepsilon_{\infty}$ values in Table 2). The value of the static dielectric constant $\varepsilon_{s}$ is found to be increasing linearly with a slope around 11.6 (per 1\% of SWCNTs) and intercept on $y$-axis of 66.36 for zero concentration of SWCNTs.

The average relaxation time obtained using Eq. (6) is found to be around $10.4 \mathrm{ps}$ and it is approximately invariant with the addition of SWCNTs as demonstrated in Table 2. One can explain this invariance of the dielectric parameters of $\varepsilon_{\infty}$ and $\tau$ due to the large contribution of the phantom material relative to the dopant material which is around $0.6 \%$ at maximum. The invariance in the relaxation time can be explained by the fact that Eq. (6) provides the average relaxation time of many relaxation processes taking place in the samples. Such relaxation processes include the relaxation time of free water, bound water and the protein in the gelatin along with the SWCNTs contribution. ${ }^{29,30}$ Since the 
Table 2. Dielectric parameters as obtained from Eq. (6) for different concentrations of SWCNTs.

\begin{tabular}{lccccccc}
\hline & $0 \%$ & $0.5 \%$ & $0.10 \%$ & $0.15 \%$ & $0.20 \%$ & $0.40 \%$ & $0.60 \%$ \\
\hline$\varepsilon_{\infty}$ & $4.9 \pm 0.2$ & $4.9 \pm 0.2$ & $4.9 \pm 0.2$ & $4.9 \pm 0.2$ & $4.9 \pm 0.2$ & $5.0 \pm 0.2$ & $4.8 \pm 0.3$ \\
$\varepsilon_{s}$ & $66.0 \pm 0.1$ & $66.9 \pm 0.1$ & $66.7 \pm 0.1$ & $69.2 \pm 0.1$ & $68.9 \pm 0.1$ & $70.9 \pm 0.1$ & $73.0 \pm 0.1$ \\
$\tau(\mathrm{ps})$ & $10.30 \pm 0.02$ & $10.47 \pm 0.01$ & $10.36 \pm 0.01$ & $10.41 \pm 0.01$ & $10.42 \pm 0.01$ & $10.40 \pm 0.01$ & $10.40 \pm 0.01$ \\
$\alpha$ & $0.088 \pm 0.001$ & $0.114 \pm 0.001$ & $0.109 \pm 0.002$ & $0.114 \pm 0.001$ & $0.117 \pm 0.001$ & $0.104 \pm 0.001$ & $0.110 \pm 0.001$ \\
\hline
\end{tabular}

concentration of SWCNTs is very small in the phantom, its contribution in the relaxation process is minimal. In order to be able to isolate the relaxation time due to the addition of SWCNTs, a wider frequency range is needed. With a wider frequency range, Eq. (2) can be used to find the dielectric parameters for the different relaxation process after the subtraction of ionic conductivity contribution from the imaginary dielectric constant. ${ }^{26}$

\section{Conclusions}

In this study, the fabricated SWCNTs-based phantom samples are found to be in a good homogeneity and the SWCNTs are dispersed well in the samples as indicated by the obtained SD. Also, it has been confirmed that addition of SWCNTs can enhance the dielectric constants (real $\varepsilon^{\prime}$ and imaginary $\left.\varepsilon^{\prime \prime}\right)$ of phantoms as seen in Fig. 3. However, when the Cole-Cole model is used to obtain the dielectric parameters (e.g., $\varepsilon_{\infty}, \varepsilon_{s}$ and $\tau$ ), the parameters are found to be invariant with SWCNTs addition, except for the $\varepsilon_{s}$ parameter which increases linearly with increasing SWCNTs concentration. The obtained average relaxation time is found to be approximately invariant with the addition of SWCNTs for the concentrations used in this study. The invariance of the average relaxation time could be attributed to the use of Cole-Cole fit that yields the average relaxation time of the $(N)$ different reorientational processes (each has its $\tau_{m}$ and its dielectric strength $\left.\Delta \varepsilon_{m}\right) \cdot{ }^{20}$ Finding the exact values of $\tau_{m}$ and $\Delta \varepsilon_{m}$ for each process is found to be a difficult task due to the limitation in the used frequency range and due to the unavoidable contribution of the salt ions in the gelatin at low frequencies.

Finally, it is important to point out that the dielectric parameters of the fabricated phantom in this study can mimic the measured dielectric parameters of real human muscles and the gray matter (gray matter is a major component of the central nervous system). ${ }^{17,31}$ From the medical applications point of view, knowing the dielectric parameters of such phantom that is doped with SWCNTS may help in developing new methods of detection and treatment of cancer-infected tissues. ${ }^{13}$

\section{Acknowledgment}

This work was funded by the Deanship of Scientific Research at Mutah University.

\section{References}

${ }^{1}$ S. Fiorito, Carbon Nanotubes: Angels or Demons? 1st edn. (Pan Stanford Publishing Singapore, 2008).

${ }^{2}$ M. Meyyappan, Carbon Nanotubes: Science and Applications (CRC Press LLC, USA, 2005).

${ }^{3}$ E. Vázquez and M. Prato, Carbon nanotubes and microwaves: Interactions, responses, and applications, ACS Nano 3, 3819 (2009).

${ }^{4}$ V. Amenta and K. Aschberger, Carbon nanotubes: Potential medical applications and safety concerns WIREs nanomed, Nanobiotechnology 7, 371 (2015).

${ }^{5}$ Z. Liu, S. Tabakman, K. Welsher and H. Dai, Carbon nanotubes in biology and medicine: In vitro and in vivo detection, imaging and drug delivery, Nano Res. 2, 85 (2009).

${ }^{6}$ B. D. Che, B. Q. Nguyen, L. T. Nguyen, H. T. Nguyen, V. Q. Nguyen, T. V. Le and N. H. Nguyen, The impact of different multiwalled carbon nanotubes on the X-band microwave absorption of their epoxy nanocomposites, Chem. Cent. J. 9(10), (2015).

${ }^{7}$ W. Shao, P. Arghya, M. Yiyong, L. Rodes and S. Prakash, Carbon nanotubes for use in medicine: Potentials and limitations, Syntheses and Applications of Carbon Nanotubes and their Composites, ed. S. Suzuki (InTech, Croatia, 2013).

${ }^{8}$ A. Sanginario, B. Miccoli and D. Demarchi, Carbon nanotubes as an effective opportunity for cancer diagnosis and treatment, Biosensors 7, 9 (2017).

${ }^{9}$ C. J. Gannon, P. Cherukuri, B. I. Yakobson, L. Cognet, J. S. Kanzius, C. Kittrell, R. B. Weisman, M. Pasquali, H. K. Schmidt, R. E. Smalley and S. A. Curley, Carbon nanotube-enhanced thermal destruction of cancer cells in a noninvasive radiofrequency field, Cancer 110, 2654 (2007).

${ }^{10}$ Y. Zhou, Mechanism and the effect of microwave-carbon nanotube interaction, Dissertation prepared for the degree of Doctor of Philosophy, University of North Texas (2005), pp. 1-78.

${ }^{11}$ D. R. Bauer, X. Wang, J. Vollin, H. Xin and R. S. Witte, Broadband spectroscopic thermoacoustic characterization of singlewalled carbon nanotubes, J. Spectrosc., 7, 2352 (2015).

${ }^{12}$ A. Mashal, B. Sitharaman, J. H. Booske and S. C. Hagness, Towards contrast-enhanced microwave-induced thermoacoustic imaging of breast cancer: An experimental study of the effects of microbubbles on simple thermoacoustic targets, Phys. Med. Biol. 54, 641 (2009).

${ }^{13}$ A. Mashal, B. Sitharaman, X. Li, P. K. Avti, A. V. Sahakian, J. H. Booske and S. C. Hagness, Toward carbon-nanotube-based theranostic agents for microwave detection and treatment of breast cancer: Enhanced dielectric and heating response of tissuemimicking materials, IEEE Trans. Biomed. Eng. 57, 1831 (2010).

${ }^{14}$ A. Mashal, F. Gao and S. C. Hagness, Heterogeneous anthropomorphic phantoms with realistic dielectric properties for microwave breast imaging experiments, Microw. Opt. Technol. Lett. 53, 1896 (2011). 
${ }^{15}$ F. Gao, S. X. Xie, B. Sitharaman, J. H. Booske and S. C. Hagness, Functionalized carbon nanotube theranostic agents for microwave diagnostic imaging and thermal therapy of tumors, IEEE Proc. 8th European Conf. Antennas and Propagation (EuCAP) (The Hague, The Netherlands, 2014) pp. 691-693.

${ }^{16} \mathrm{M}$. Raoof and S. A. Curley, Non-invasive radiofrequency-induced targeted hyperthermia for the treatment of hepatocellular carcinoma, Int. J. Hepatol. 2011 (2011).

${ }^{17}$ M. Lazebnik, E. L. Madsen and S. C. Hagness, Tissue-mimicking phantom materials for narrowband and ultra-wideband microwave applications, Phys. Med. Bio. 50, 4245 (2005).

${ }^{18}$ A. Abu Bakari, A. Abboshi, P. Sharpe and M. Bialkowskii, Artificial breast phantom for microwave imaging modality, IEEE EMBS Conf. Biomedical Engineering \& Sciences (Kuala Lumpur, Malaysia, 2010), pp. 385-388.

${ }^{19}$ M. S. M. Said, N. Seman, M. K. A. Rahim and T. A. Rahman, Investigation on dielectric properties in gelatin-based phantom for human brain, IEEE Proc. ISAP 2014 (Kaohsiung, Taiwan, 2014), pp. 585-586.

${ }^{20}$ G. G. Raju, Dielectrics in Electric Fields, 2nd edn. (Taylor \& Francis, CRC, USA, 2017).

${ }^{21}$ S. Havriliak and S. Negami, A complex plane analysis of alphadispersions in some polymers, J. Polym. Sci. C: Polym. Symp.14, 99 (1966).

${ }^{22}$ L. Mariya, Ultra wide band spectroscopy and dielectric-properties contrast enhancement, Dissertation, University of WISCONSIN MADI-SON (2008).

${ }^{23} \mathrm{D}$. Xu, L. Liu and Z. Jiang, Measurement of the dielectric properties of biological substances using an improved open-ended coaxial line resonator method, IEEE Trans. Microw. Theory Tech. 35, 1424 (1987).
${ }^{24}$ S. A. Komarov, A. S. Komarov, D. G. Barber, M. L. Lemes and S. Rysgaard, Open-ended coaxial probe technique for dielectric spectroscopy of artificially grown sea ice, IEEE Trans. Geosci. Remote Sens. 54, 4941 (2016).

${ }^{25}$ D. Misra, M. Chabbra, B. R. Epstein, M. Mirotznik and K. R. Foster, Noninvasive electrical characterization of materials at microwave frequencies using an open-ended coaxial line: Test of an improved calibration technique, IEEE Trans. Microw. Theory Tech. 38, 8 (1990).

${ }^{26} \mathrm{M}$. Wolf, R. Gulich, P. Lunkenheimer and A. Loid, Relaxation dynamics of a protein solution investigated by dielectric spectroscopy, Biochim. Biophys. Acta 1824, 723 (2012).

${ }^{27}$ Dielectric Probe Kit $10 \mathrm{MHz}$ to $50 \mathrm{GHz}$ Technical Overview. Keysight Technologies, 2015-2018 (USA, February 8, 2018). https://literature.cdn.keysight.com/litweb/pdf/5992-0264EN.pdf? id $=2605692$.

${ }^{28}$ S. W. Lan, M. H. Weng, R. Y. Yang, S. J. Chang, Y. S. Chung, T. C. Yu and C. S. Wu, Preparation of a carbon doped tissue-mimicking material with high dielectric properties for microwave imaging application, Materials, 9, 559 (2016).

${ }^{29}$ A. V. Vorst, A. Rosen and Y. Kotsuka, RF Microwave Interaction with Biological Tissues (John Wiley \& Sons, Canada, 2006).

${ }^{30}$ V. Arvind, V. Sarode, R. N. Gacche and A. C. Kumbharkhane, Non-invasive radiofrequency-induced targeted hyperthermia for the treatment of hepatocellular carcinoma, RSC Adv. 4, 40711 (2014).

${ }^{31}$ S. Gabriel, R. W. Lau and C. Gabriel, The dielectric properties of biological tissues: III. Parametric models for the dielectric spectrum of tissues, Phys. Med. Biol. 41, 2271 (1996). 